\title{
Intelligent learning and testing system for students training in the problem area of nanotechnology and microsystem engineering
}

\author{
D. Lyapunov ${ }^{1,4}$, A. Yankovskaya ${ }^{1,2,3,5}$, Y. Dementyev ${ }^{1}$, K. Negodin ${ }^{1}$ \\ ${ }^{I}$ National Research Tomsk Polytechnic University, 30, Lenin Ave., 634050, Tomsk, Russia \\ ${ }^{2}$ National Research Tomsk State University, 36, Lenin Ave., 634050, Tomsk, Russia \\ ${ }^{3}$ Tomsk State University of Control Systems and Radioelectronics, 40, Lenin Ave., 634050, Tomsk, Russia \\ ${ }^{4}$ Research Intsitute of Automation and Electromechanics, 53, Belinskiy St., 634034, Tomsk, Russia \\ ${ }^{5}$ Tomsk State University of Architecture and Building, 2, Solyanaya Sq., 634003, Tomsk, Russia
}

\begin{abstract}
For the students learning and training in the field of nanotechnology and microsystem engineering within the framework of blended learning paradigm we need high quality content; efficient learning technology; means of students motivation; evaluation tools. We propose an intelligent learning and testing system based on mixed diagnostic tests for effective comprehension of a number of subjects within the problem area. The system allows to provide effective comprehension of a number of subjects and to form the primary competences from the students point of view, revealing their future occupation preferences. During the learning process the students within small groups (not less than 4 students) solve the problems of development, modelling and design of microsystem devices. They also investigate the market needs, consider the opportunities of macroscopic sensors and actuators exchange on their microsystem analogs.
\end{abstract}

Keywords: Intelligent learning and testing system; nanotechnology; microsystem engineering; learning technology; blended learning; mixed diagnostic test; competences; multidisciplinary course

\section{Introduction}

A relevance of intelligent learning and testing systems (ILTS) design for students training in different problem areas is well acknowleged [1]. Currently the need of ILTS in such interdisciplinary field as nanotechnology and microsystem engineering is justified by including the field in the list of critical technologies of the Russian Federation [2]. Domestic industrial enterprises need highly qualified specialists in the problem area. The need is caused by the rapid development of nanotechnologies and the progress of new metamaterials fabrication. The materials under consideration can possess unique mechanical, electrical, magnetic, thermal and optical properties [3].

Current trends, challenges and news in the problem area are published in the Journal of Nano- and Microsystem Engineering [4] and in a number of foreign journals.

The specialty education on the program "Nanotechnology and Microsystem Engineering" is provided in such Russia's privileged institutions as Moscow State Technical University of Radioengineering, Electronics and Automation, Kazan Federal University, Saint-Petersberg State Polytechnic University, Tomsk State University of Control Systems and Radioelectronics, Siberian Federal University and others.

We should note that the problem area "Nanotechnology and Microsystem Engineering" is a multidisciplinary one. Therefore, the learner should possess the competences in the following disciplines: chemistry, physics, material science, electrical engineering, electronics, thermal engineering, optics, mathematics, and others. These competences are essential for research of nano- and microscale phenomena taking into account the latest technological achievements.

For effective learning and training in the problem area the students need: high quality online content; effective learning technologies; means of motivation; cognitive graphic tools (CGT) for learning outcomes evaluation and timely feedback at each state of learning process.

We propose an intelligent learning and testing system (ILTS) based on mixed diagnostic tests (MDT), aimed at effective comprehension of a number of disciplines in the problem area of nanotechnology and microsystem engineering. Moreover, the learner will be able to construct an individual learning trajectory, to enhance his/her strengths, and fill the knowledge gaps.

\section{Peculiarities of the problem area "Nanotechnology and Microsystem Engineering"}

Before we go any further, we consider the inherent features of the problem area. Most essential of them are:

1) Rapid development of nanotechnologies, microsystem components modelling and design methods.

2) Specialists orientation primarily in scientific research.

3) Lack of standards on some nanotechnology materials and products.

4) Microsystem components miniaturization trend (Moor's law is still valid).

To increase the industrial release of nanotechnology products we should:

1) provide advanced training of the students and the specialists in the problem area;

2) design specialized equipment for nanomaterials and microsystem products fabrication;

3) fulfill a database on modern materials and microsystem constructions;

4) design the testing devices for checking industrially released microsystems. 
Semi-empirical engineering methods of modelling and design, borrowed from the adjacent disciplines (material science, electrical engineering, electronics, thermodynamics, and others), should be supplemented by new methods of microsystem devices modelling and design [5]. Using the methods the future specialists will be able to construct promising microsystem devices, taking into account detailed structural analysis of the object under development, regularities of its response, prediction of its functional characteristics.

Students learning endeavor in any field of interest, especially when we deal with such field as nanotechnology, is primarily connected with their motivation to comprehend the learning course completely. The need of students' motivation is described in detail in [6,7].

A goal setting performed by the student contributes greatly the motivation issues. An effective goal setting is proved to be the guaranteed comprehension of the discipline under study, thus moving towards the goal [6]. Unfortunately, the learning outcomes declared in the syllabi of the majority of disciplines do not correspond to the students personal goals and preferences. Therefore, at the initial stage of the learning process we must provide students with high quality lecture material along with online content, aimed to increase students interest. It can help to correct students personal goals at the early stage of the learning process. Thus, the students will be motivated to implement systematic and progressive steps to master the discipline under study.

We develop the ILTS for the advanced mastering the discipline taking into account the individual features and needs of each learner and the peculiarities of the problem area of nanotechnology and microsystem engineering.

\section{Basic Terms and Definitions}

Herein we introduce some terms and definitions used in the future chapters of the paper.

A learner is a human who is learning, e.g. the University student.

A teacher is a human who is managing and/or supporting the learning process.

Diagnostic test (DT) is a set of features differentiating any pairs of objects, which belong to different patterns.

Unconditional diagnostic test (UDT) is characterized by simultaneous presentation of all the intrinsic features (questions) of the object under study during the decision-making process.

Conditional diagnostic test (CDT) is a test, in which each subsequent feature (question) depends on the previous features (questions) represented to a learner.

Mixed diagnostic test being a compromise between unconditional and conditional components [8].

\section{Basics of Intelligent Learning and Testing Intelligent System Construction}

We have been developing the ILTS since late 2010th [1,8,9]. An evolution of knowledge acquired during a learning course, represented by a semantic web, was introduced in the paper [9]. We demonstrate the block diagram of the ILTS in Fig. 1.

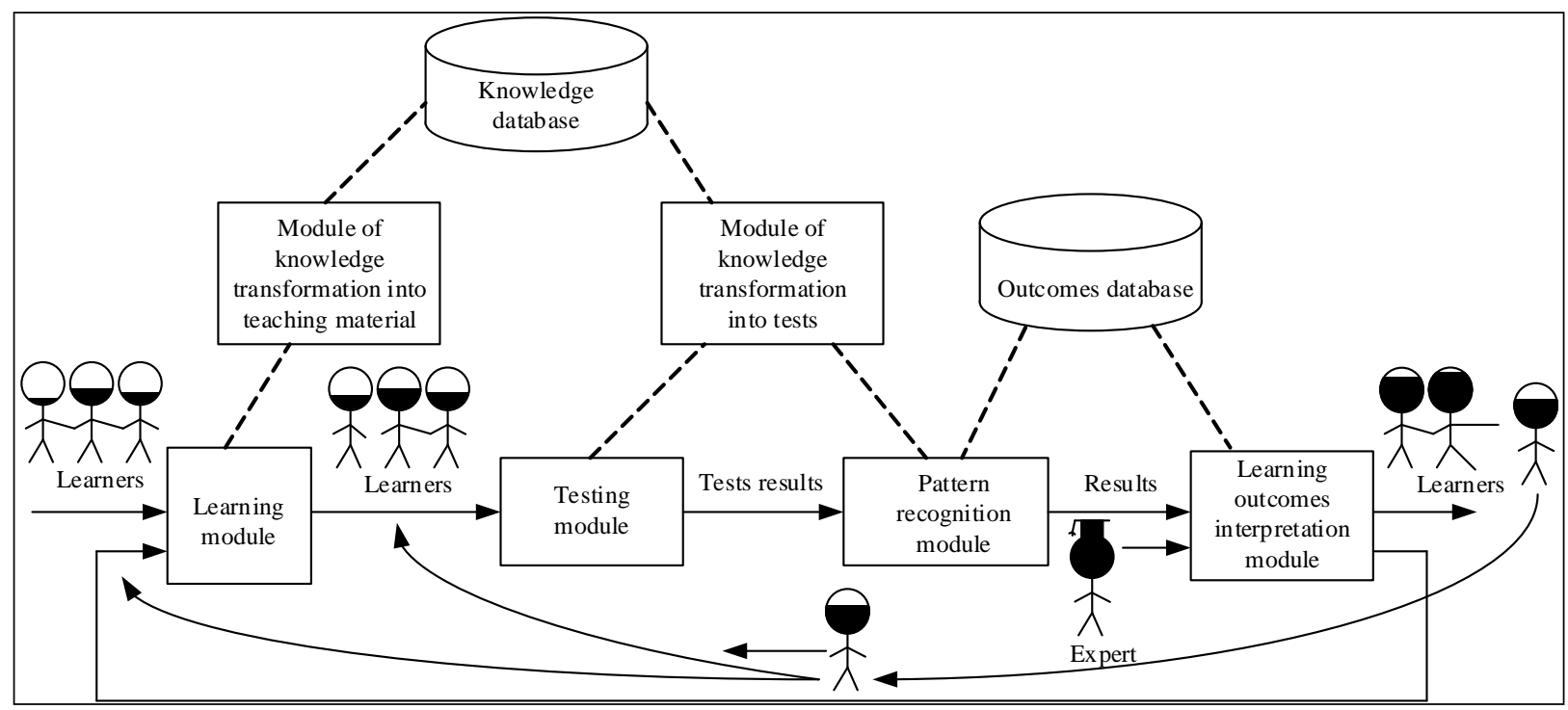

Fig. 1. A block diagram of the intelligent learning and testing system.

The algorithm of ILTS we subdivided into 7 subsequent steps.

Step 1. During a learning process a student comprehends the learning materials subsequently. The learning materials on a particular topic are represented by a text with an interactive multimedia content. We use the semantic model of knowledge representation to store the data while learning [9]. Learning module is responsible for this step.

Step 2. The ILTS acquires and stores the results of UDT. Steps 2-4 are performed via testing module.

Step 3. If the learner succeeded in the UDT, ILTS switches him/her to CDT. In this case the system defines each subsequent question depending on the answer on the previous one. 
Computer Optics and Nanophotonics / D. Lyapunov, A. Yankovskaya, Y. Dementyev, K. Negodin

Step 4. The results of all MDT components (UDT and CDT) are recorded in the results database. We use high level of detalization to get additional data for the learning course improvement in the future. The ILTS generates Learner Action Card (LAC) for each student.

Step 5. After each learning module the LAC is converted into a set of evaluation indicators, such as: a) theory knowledge level; b) problems solving skills; c) laboratory work performance evaluation. Thus, the ILTS suggests the future learning trajectory for the student using pattern recognition module.

Step 6. After finishing the learning course the ILTS represents to each student the evaluated knowledge, LAC interpretation and evaluation indicators calculated. Students compare the learning materials comprehended with knowledge and competences acquired during the course. They reveal their knowledge gaps and a lack of skills by concurrent consideration of the LAC and the semantic web of the learning course. If there is some revealed knowledge gap or a lack of competence in the problem area, the student has an option to return to the step 1 and to practice learning materials and tests one more time. In this case the learner is involved in the decision-making process, based on analyzing of the learning outcomes via cognitive graphic tools (CGT) and revealing the knowledge gaps and the future challenges. As a result, the student constructs his/her individual learning trajectory. The present step is provided by learning interpretation module.

Step 7. When the testing procedure is finished, the learner is considered to comprehend the entire learning course as well as interdisciplinary connection between the course modules. In this case the ILTS calculated the total evaluation expressing the mean value of all the tests results.

We organized the sequence of the learning material representation so as each subsequent learning module is connected with the previous one. Each learning module is designed in accordance with a reflection cycle [10], represented in Fig. 2 by a directed cyclic diagram.

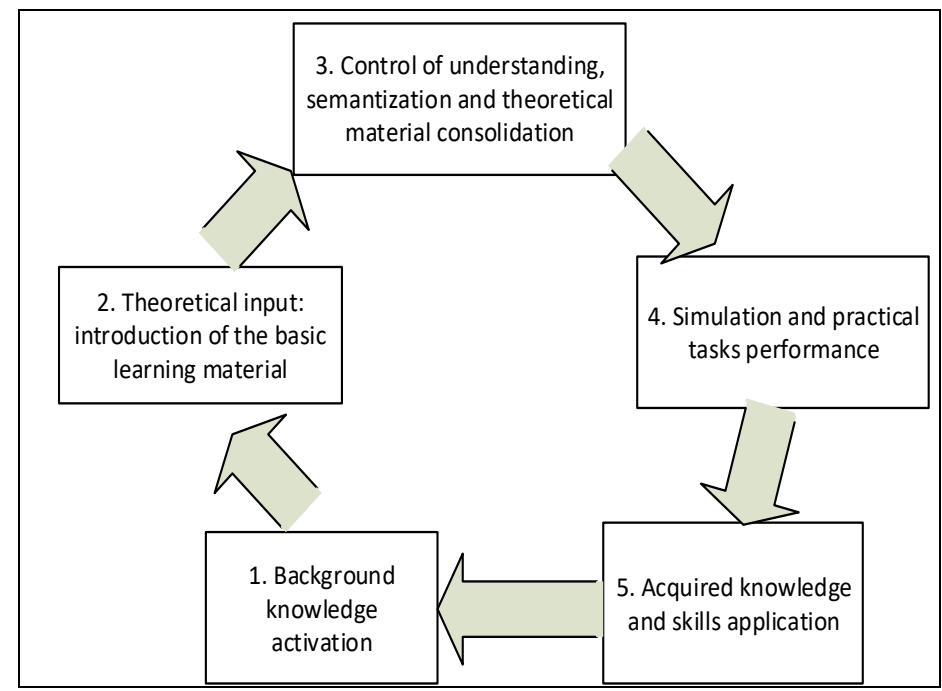

Fig. 2. Reflection cycle.

\section{Learning Outcomes Interpretation}

In this chapter we use some fragments from the papers [11,12], wherein the usage of 2-simplex prism CGT is entirely described for students' learning outcomes interpretation. We have used the advantages of the 2-simplex prism for students performance evaluation during the course "Selected Chapters of Electronics", which includes the module "Microelectromechanical Systems". An example of individual learning trajectory construction based on the MDT and 2-simplex prism CGT is shown in Fig. 3.

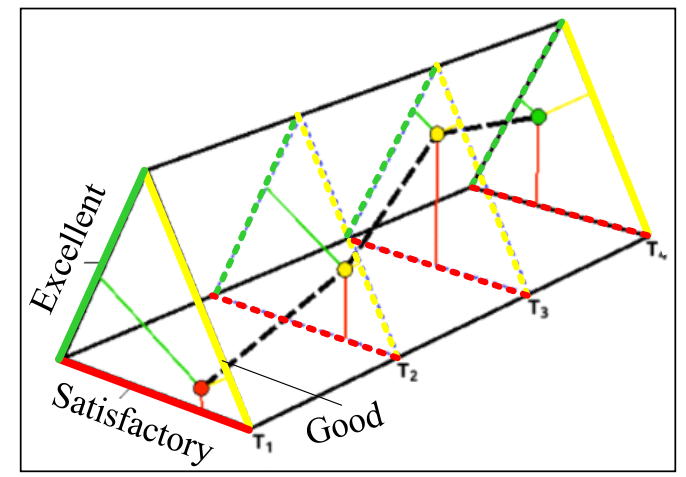

Fig. 3. Learning outcomes evaluation using 2-simplex prism cognitive graphic tool.

The results of each of the four tests are represented as points (the small circles of different colors) within 2-simpleces (crosssections of the 2-simplex prism). Prism's faces correspond to evaluation indicators (grades): 1) "excellent"; 2) “good"; 3) "satisfactory". The distance from the base of the 2-simplex prism to the 2-simplex (equilateral triangle) under consideration 
corresponds to the time interval from the beginning of the learning process to the time of the corresponding testing. The dashed line within the 2-simplex prism shows the knowledge level evolution of the student based on the test results at the time moments T1, T2, T3 and T4. We note that illustrations presented in Fig. 3 and 4 were obtained using visualization library, which is currently under development and is available via the link [13].

We observe in Fig. 3 that the student had got a grade close to "satisfactory" at the input testing at time moment T1. Although the grade is between "satisfactory" and "good". Then, having analyzed the test result, the student had set a goal to increase his/her evaluation indicators. During the 2nd testing the learner demonstrated better result (the grade is close to "good") at time moment T2. Then, at time moment T3 the testing result corresponds to the intermediate grade between "good" and "excellent". And, finally, at time moment T4 the student achieved the goal, which he/she had set on the previous stages of the learning process (the grade is close to "excellent").

Desired area of development of the learner is chosen based on the MDT results represented by CGT 2-simplex. In Fig. 4 we represent the 2-simplex CGT. The patterns in the form of points (the circles of small radii of different color) correspond to competences of the four students after finishing the learning course.

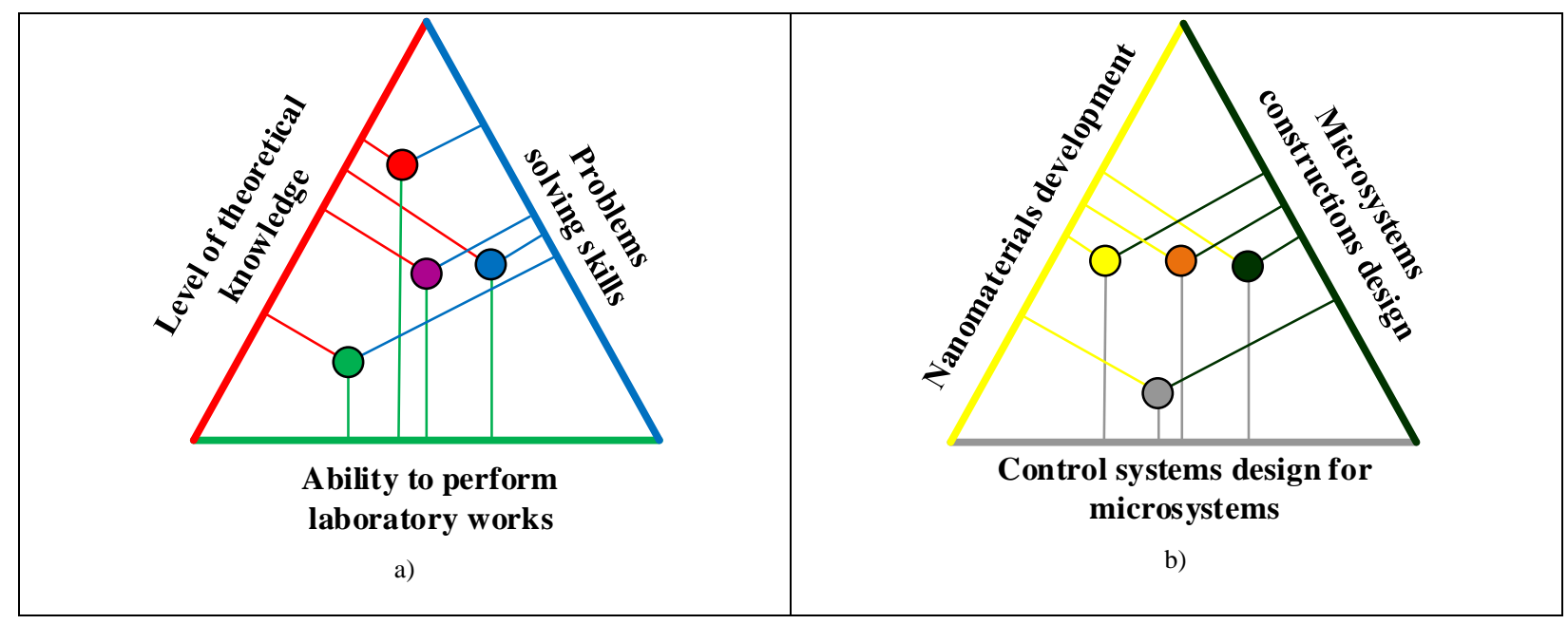

Fig. 4. 2-simpleces indicating the competences of the four students after finishing the learning course: a) according to knowledge, skills and abilities; b) according to individual aptitude to future occupation.

Each point in Fig. 4 corresponds to the balance state between the competences gained (level of knowledge, problems solving skills and ability to perform laboratory works) of the one learner. Consider the perpendicular connecting the point under study (e.g. the green one) and the side of the equilateral triangle (e.g. the side, which relates to the ability to perform laboratory works). The length of the perpendicular (green line) characterizes the students' ability under consideration. The smaller is this length, the higher is the student's evaluation of the corresponding competence. The green point (see Fig. 4, a) corresponds to testing results of the student, who gained the laboratory works performance ability in the best way, whose theoretical knowledge is quite good, but the problems solving skills are lacking. The red point characterizes the student, who knows the theory and gained problems solving skills at sufficient level, but whose ability to perform the laboratory works is not sufficient. The blue point expresses the testing results of the student who copes with problem solving in the best way, but needs improving in other areas. The violet point corresponds to the testing results of the student who achieved a balance between the competences under consideration.

Considered competences evaluations are the basis for further goal setting, taking into account all strong points and weaknesses of a particular student. The ILTS is aimed to reveal the gaps in competences and to propose the actions, which will help to eliminate them in the future.

We illustrate the testing results of the four students in Fig. 4, b. The test was constructed to reveal individual aptitude towards future occupation. Each point shown in Fig 4, b demonstrates the aptitude to the future occupation for each of four students. The yellow point corresponds to the testing result of the student, who is apt to conduct research in the field of material science to get the new materials. The dark green point corresponds to the learning outcomes of the student, who is good in microsystems constructions design. The grey point corresponds to the aptitude of microsystem devices control systems design. Finally the orange point corresponds to the student who achieved a balance between the components and have wide range of aptitudes.

Test results analysis gives an information, which is used to construct a learning trajectory. It facilitates the competence improvement in such areas as: 1) scientific research aimed at new technologies development of microsystems fabrication; 2) practical activities in the field of microsystems design; 3) teaching in the nanotechnology and microsystem engineering problem area.

\section{Conclusion}

Intelligent Learning and Testing System (ILTS) proposed is designed for the learning process efficacy increase in such multidisciplinary fields as nanotechnology and microsystem engineering, and also in the data analysis research. Learning outcomes analysis will allow to effectively design the learning courses. The courses under development are based on the mixed diagnostic tests. By using the ILTS we take into account individual peculiarities of the students during their learning activities, 
Computer Optics and Nanophotonics / D. Lyapunov, A. Yankovskaya, Y. Dementyev, K. Negodin

reveal different types of regularities in the learning process, correspond the learner's individual preferences and learning outcomes. The ILTS provides a balanced learning material representation on every stage of learning process.

We use the semantic technologies to compare the learning material represented and the knowledge acquired for each learner providing filling the knowledge gaps if necessary. The feedback at every stage of learning process in the form of semantic web provides timely correction and repetition of uncomprehended material. In addition, the student reveals his/her field of competence, i.e. the most "sweet" course modules, thus, constructing the individual learning trajectory.

To the present time, a number of modules on the topic "Microsystem Engineering" were implemented into the e-learning course "Selected Chapters of Electronics". The learning outcomes of the students, who participated in the course, give grounds to believe that using the ILTS proposed we are able to motivate students to achieve the goals set by them in the early stages of the learning process. We propose the use of ILTS for bachelor training program "Nanotechnology and Microsystem Engineering" for disciplines: "Introduction to Nanotechnology", "Microsystem Technology", "Microelectromechanical Systems", "Digital Control Systems" and others.

\section{Acknowledgements}

The research was funded by RFBR grant (project No. 16-07-00859a). The authors are grateful to the junior research fellow, assistant of the Tomsk State University of Control Systems and Radioelectronics, Artem V.Yamshanov for the cognitive graphic tools software design and implementation for the data analysis. We are also grateful to the $3^{\text {rd }}$ year students (group 5G4B) major in Electric Power Engineering who participated in the learning course "Selected Chapters of Electronics" for their patience, persistence and desire to learn.

\section{References}

[1] Yankovskaya AE, Semenov ME, Yamshanov AV, Semenov DE. Gognitive means in learning and testing systems based on mixed diagnostic tests. Artificial Intelligence and Decision Making 2015; 4: 51-61.

[2] A list of critical technologies of the Russian Federation. URL: http://www.kremlin.ru/supplement/988 (01.02.2017).

[3] Galochkin V. Introduction to nanotechnology and nanoelectronics. Samara: Povolzhsky State University of Telecommunications and Informatics, 2013; 367 p. (in Russian).

[4] Interdisciplinary, scientific, technique and production journal "Nano- and Microsystems Technology". URL: http://www.microsystems.ru/ (02.02.2017).

[5] Lyapunov D. MEMS capacitance converters: Constructions, materials, research issues. Saarbrucken : LAP LAMBERT Academic Publishing GmbH \& Co. KG, 2012; 139 p. (inRussian).

[6] Dirksen J. Design for how people learn. Berkeley: New Readers, 2012; 272 p.

[7] LeFever L. The Art of Explanation. New Jersey: Wiley and Sons, 2012; 378 p.

[8] Yankovskaya AE. Design of Optimal Mixed Diagnostic Test With Reference to the Problems of Evolutionary Computation. Proceedings of the 1st International Conference on Evolutionary Computation and Its Applications, Moscow, 1996; 292-297.

[9] Yankovskaya AE, Shurygin YuA, Yamshanov AV, Krivdyuk NM. Determination of the level of acquired knowledge on the training course presented by the semantic network. Proceedings of International Conference“Open Semantic Technologies for Intelligent Systems” (OSTIS-2015), Minsk, 2015; 331-338.

[10] Plekhanova MV, Prohorets EK. Modeling of electronic courses based on the reflective cycle. International Journal of Applied and Fundamental Research 2015; 5: 600-604.

[11] Yankovskaya AE, Yamshanov AV, Krivdyuk NM. 2-simplex Prism - a Cognitive Tool for Decision Making and Its Justification in Intelligent Dynamical Systems. Machine Learning and Data Analysis 2015; 1(14): 1930-1938.

[12] Yankovskaya AE, Dementyev YuN, Lyapunov DY, Yamshanov AV. Intelligent Information Technology in Education. Information Technologies in Science, Management, Social Sphere and Medicine (ITSMSSM-2016). Atlantis Press Publishing 2016; $17-21$.

[13] 2-simplex Prism Cognitive Graphic Tool. URL: http://cogntool.tsuab.ru/ 250 demos/2-simplex-prediction/ (02.02.2017). 